\title{
Management of congenital diaphragmatic hernia
}

Congenital diaphragmatic hernia $(\mathrm{CDH})$ has an incidence of between one in 2000-5000 live births. The hidden mortality associated with $\mathrm{CDH}$ includes spontaneous abortions and stillbirths as well as therapeutic abortions (often for other identified anomalies). The management of $\mathrm{CDH}$ has been intensely debated since Ladd and Gross stated in 1940 that early surgery was the proper treatment for these patients. ${ }^{1}$ Controversies have surrounded ideal preoperative management, timing of surgery, and indeed whether surgery should be offered to all. ${ }^{2}$ Despite the advances in neonatal care, inroads into mortality which might have been anticipated have not materialised and it remains relatively unchanged at $40-50 \% .^{3}$ However, we now stand on the threshold of an exciting stage in the development of neonatal cardiorespiratory support. The role of new therapeutic modalities such as extracorporeal membrane oxygenation (ECMO), surfactant, high frequency ventilation (HFV), inhaled nitric oxide (iNO), in utero interventions, and liquid ventilation have intensified the debate.

\section{Antenatal management}

Prenatal diagnostic rates vary from 10-90\% depending on local antenatal ultrasound screening protocols. Prenatal diagnosis allows for counselling of parents and planned management but does not predict outcome. ${ }^{4}$ Prenatal diagnosis of $\mathrm{CDH}$ should lead to careful inspection for other major anomalies and chromosomal analysis is justified. Laterality, presence of liver and stomach in chest, left ventricular mass, presence of polyhydramnios, and ultrasound indices of relative lung volume have been used in attempts to predict outcome, but are not reliable. Only the presence of associated major anomalies is reliably associated with a poor prognosis. ${ }^{5}$

IN UTERO SURGERY

To date, a number of surgical approaches have been attempted. After careful preparatory animal studies, Harrison et al repaired the defect by fetal surgery. ${ }^{6}$ Results were disappointing as the technique could only be used in those without liver herniation. Even with technical success, there was a poor outcome because of preterm labour. Based on the observation that an obstructed trachea prevents the development of lung hypoplasia, ${ }^{7}$ a number of techniques have been devised on the "plug the lung until it grows" principle. ${ }^{8}$ These include a variety of tracheal plugs and tracheal clipping at fetoscopy. Encouraging results have been reported in experimental settings. Clinical results are preliminary but there remain many obstacles. ${ }^{9}$ Premature labour is a major problem with all in utero techniques. Outcomes have been generally disappointing.

\section{PERINATAL CARE}

Close liaison between the fetal medicine/obstetric, neonatal, and surgical teams is essential. The infant with prenatal diagnosis of $\mathrm{CDH}$ should be delivered in an institution with full facilities and a management strategy. Antenatal glucocorticoid administration, now standard practice in the prevention of respiratory distress syndrome after premature labour, has been shown to improve pulmonary immaturity in rats with experimentally induced $\mathrm{CDH}$ and a small study using low dose dexamethasone and thyrotrophin releasing hormone showed benefit in patients. ${ }^{10}$ A larger, prospective study is needed. Senzaki and colleagues have proposed the administration of morphine and pancuronium in the perinatal period to prevent the proposed enlargement of the hernia and mediastinal deviation caused by spontaneous breathing just after birth as an adjunct to immediate perinatal management. ${ }^{11}$

The EXIT technique, whereby the baby is delivered through the hysterotomy at caesarean section, allows interventions such as immediate intubation and delivery of surfactant before the first breath to be undertaken while the fetus is partially delivered but still supported by the placental circulation. This is an approach presently being evaluated.

\section{Postnatal management}

The major underlying problems in $\mathrm{CDH}$ are pulmonary hypoplasia and high pulmonary vascular resistance which cause right-to-left shunting of blood away from the lungs and failure of adaptation to extrauterine life. This leads to hypoxia, hypercarbia, and acidosis, all of which exacerbate pulmonary hypertension. Management strategies are structured to optimise gas exchange and to reduce pulmonary hypertension while minimising ventilator induced lung damage.

IMMEDIATE CARE

A high index of suspicion is necessary to establish postnatal diagnosis. The presence of a scaphoid abdomen, deviation of the heart beat, and unilateral diminished breath sounds in the presence of respiratory distress prompt the diagnosis. The infant should be intubated immediately after birth. It is important to avoid mask ventilation which will exacerbate respiratory embarrassment. A large bore gastric tube (minimum $10 \mathrm{FG}$ ) is passed to decompress the intrathoracic intestine. Contralateral pneumothorax is a recognised complication of over vigorous resuscitation.

TRANSPORTATION

For those infants born outside a tertiary centre, safe transport to a specialised paediatric surgery unit is essential. This requires pretransfer stabilisation, experienced transfer staff, full monitoring facilities, and a range of treatment options.

VENTILATION STRATEGIES

Gentle ventilation is critical and conventional ventilator parameters should be set to minimise barotrauma in a setting of bilateral hypoplastic, delicate lungs. The aim is to achieve adequate oxygenation with the lowest possible pressures. Data from preductal and postductal saturation monitors and a preductal arterial line should be used to optimise ventilation. A preductal $\mathrm{pH} \geqslant 7.20$ and an arterial oxygen pressure of $\geqslant 6.5 \mathrm{kPa}$ (with an oxygen saturation of 85-90\%) should be accepted. Permissive hypercapnia will reduce ventilation induced lung damage. Excessive ventilation parameters should not be used in an attempt to normalise blood gas results. A cardiac echo will demonstrate a persistent patent ductus arteriosus and determine ductal flow. Alternative strategies may be necessary to minimise the right-to-left shunt. Adequate analgesia and 
sedation is essential. Most centres use muscle relaxants though a number advocate awake ventilation using patient triggered ventilation techniques. ${ }^{12}$ Attention to fluid balance should ensure adequate perfusion of tissues without causing pulmonary overload.

HIGH FREQUENCY VENTILATION

HFV, either initially or when conventional ventilation proves ineffective, is an attractive approach to optimise alveolar recruitment and reduce barotrauma. Most experience is of oscillatory ventilation (HFOV). The rationale is that small tidal volumes and gas transport by augmented diffusion, results in lower shear stresses and less barotrauma. Better alveolar recruitment with HFOV may also be beneficial in delivering iNO to the pulmonary resistance vessels. The place of $\mathrm{HFOV}$ in $\mathrm{CDH}$ is uncertain and prospective studies are needed. ${ }^{13}$

INTRATRACHEAL PULMONARY VENTILATION (ITPV)

ITPV is an ingenious technique developed by Theodore Kolobow, where a high gas flow is introduced into the trachea just above the carina in a reverse direction via an ultrathin endotracheal tube. It allows for excellent carbon dioxide removal and very low intratracheal ventilation pressures. Experience is very limited to date. ${ }^{14}$

\section{INTRAVASCULAR VASODILATOR AGENTS}

The search for a specific pulmonary vasodilators has so far been unrewarding. Tolazoline has histamine-like action as well as being an adrenergic antagonist with cholinomimetic properties. It has no place in the management of $\mathrm{CDH}$. Magnesium sulphate has had some reported success. It lowers smooth muscle intracellular calcium and may inhibit vasoconstriction. Prostacycline acts via cGMP and also lowers intracellular calcium. It has the disadvantage of platelet inactivation. All intravascular agents cause variable systemic hypotension. An inotrope such as dopamine may be needed to augment systemic pressure.

INHALED NITRIC OXIDE

Inhaled NO allows selective pulmonary vasodilatation. It has been shown to reduce the need for ECMO support in appropriate infants with persistent pulmonary hypertension of the newborn. ${ }^{15}$ Its value in the infant with $\mathrm{CDH}$ is less clear. Concern has been expressed over potential side effects. Its use leads to a reduction in endogenous production of NO, and in selected infants with $\mathrm{CDH}$ the response and benefit may be temporary. ${ }^{16}$

\section{SURFACTANT}

Experimental studies have revealed biochemical immaturity in association with $\mathrm{CDH} .{ }^{17}$ However, amniotic fluid analysis has not proved an indicator of surfactant deficiency in the human fetus. Administration of surfactant in experimental conditions and in neonates has revealed at best initial benefit. ${ }^{18}$ There continues to be the problem of inadequate quantity, distribution, and inactivation of surfactant in CDH. It may need to be administered prophylactically, before the first breath.

\section{EXTRACORPOREAL MEMBRANE OXYGENATION}

The utilisation of partial cardiorespiratory bypass allows for lung rest and reduction in pulmonary vascular resistance. Data from the Extracorporeal Life Support Organization (ELSO) from January 1997 on over 2450 cases reported $59 \%$ survival for neonates with $\mathrm{CDH}$ receiving ECMO support. In the UK Collaborative Neonatal ECMO Trial ECMO was significantly better than conventional management, ${ }^{19}$ though less good than the ELSO data. Surgery may be performed while on

\section{Key messages}

- Infants with $\mathrm{CDH}$ should be delivered in specialist centres

- Gentle ventilation techniques, including permissive hypercapnia to protect the delicate lungs, and strategies to reduce pulmonary hypertension should be employed

- Surgery should be delayed until pulmonary hypertension has been stabilised in utero surgery offers hope for high risk $\mathrm{CDH}$ but techniques are not yet perfected and premature labour remains a problem

- Postnatal lung growth strategy with perfluorocarbon liquid and ECMO support is encouraging for high risk cases

ECMO support, as support after $\mathrm{CDH}$ repair or repair may be performed after decannulation. It has been proposed that ECMO should be used selectively in cases where pulmonary hypertension predominates but not in cases of marked pulmonary hypoplasia. ${ }^{2}$ Others have argued that no one should be excluded from ECMO ${ }^{20}$ Lally et al concluded from their review of five ECMO centres that preoperative stabilisation with ECMO and repair on bypass may allow some high risk infants to survive. ${ }^{21}$

LIQUID VENTILATION

The clinical use of perfluorocarbon liquid ventilation has been the single most exciting recent development in respiratory intensive care since the introduction of ECMO. Studies of its use in $\mathrm{CDH}$ are preliminary. ${ }^{22}$ To date, it has not been dramatically successful in $\mathrm{CDH}$, though the use of perfluorocarbon liquid to achieve postnatal lung growth by distending the lungs is very exciting. A lung growth strategy in conjunction with ECMO support may be the optimal postnatal management in severe $\mathrm{CDH}$.

LUNG TRANSPLANTATION

Van Meurs et al have reported a case of lobar lung transplantation as a treatment for $\mathrm{CDH}$ utilising ECMO as a bridge to transplantation. ${ }^{23}$ Work has been carried out to induce immune tolerance for postnatal transplantation. This solution, however, is unlikely to be other than occasional and is not presently performed in the UK.

\section{Surgery for CDH}

Respiratory mechanics, rather than improving, often deteriorate after $\mathrm{CDH}$ repair. ${ }^{24}$ Emergency surgery has been replaced by optimisation of patient cardiorespiratory status followed by delayed surgery. Delayed surgical repair has also been used successfully in conjunction with ECMO.

An abdominal approach is best via a subcostal incision. A hernial sac, if present, is excised. Abdominal contents, often including a segment of liver and the spleen in addition to intestine, are gently returned to the abdomen. The defect is closed primarily where possible. If the defect is large, a prosthetic patch may be necessary. The authors use polytetrafluoroethylene preferentially. The ipsilateral chest should be carefully inspected to view the lung and rule out other abnormalities (for example sequestrated lobe). A chest drain is not necessary and may cause excessive mediastinal shift to the side of the repair with overdistension of the contralateral lung.

The bowel is usually not fully rotated, but, as no obstructive Ladd's bands are present, there is no indication for a Ladd's procedure and the appendix should not be removed. Rarely, it is necessary to use an abdominal prosthetic patch to obviate high intra-abdominal pressure in the early postoperative period. 


\section{Follow up}

Survivors can have a range of problems. ${ }^{25}$ Failure to thrive is a feature of many survivors of $\mathrm{CDH}$. Gastro-oesophageal reflux and oesophageal dismotility are common after $\mathrm{CDH}$ repair. Gastro-oesophageal reflux tends to be refractory to medical treatment and may require surgical management. Most survivors have adequate respiratory function, though long term ventilation has been associated with bronchopulmonary dysplasia. Some patients have thoracic deformities severe enough to warrant corrective surgery in later life. Neurodevelopmental outcome generally reflects the severity of illness and in this regard sensorineural deafness needs to be considered.

\section{The future}

The principle of gentle ventilation aimed at preventing ventilation induced lung injury is well established. Surgical repair will be delayed while the reactivity of the pulmonary vasculature is manipulated. Antenatal maternal glucocorticoid/thyrotrophin releasing hormone prophylaxis will be included in protocols if studies confirm benefit in the fetus with $\mathrm{CDH}$. There may be a place for prophylactic surfactant, given at delivery before the first breath. Better antenatal diagnosis and more accurate indicators of the high risk fetus with $\mathrm{CDH}$ who might benefit from fetal intervention will hopefully occur. As technical problems are overcome and should effective control of preterm labour be perfected, fetoscopic intervention may be the ultimate optimal management for the high risk antenatally diagnosed $\mathrm{CDH}$.

The role of iNO will be clarified by trials, safety profiles will be determined, and optimal delivery protocols will be defined. Research continues into other mediators of pulmonary resistance vessels. Recently, there has been interest in the role of 5-hydroxytryptamine and the vasoconstrictor peptide endothelin-1 in the pathophysiology of pulmonary hypertension. Specific cell surface receptor antagonists to these substances in the lung vasculature may play a part in the reversal of pulmonary hypertension in $\mathrm{CDH}$.

Venovenous ECMO, possibly in conjunction with perfluorocarbon induced lung distension, may be the optimal approach for infants with $\mathrm{CDH}$ who do not respond to less invasive management.

C F DAVIS

Department of Surgical Paediatrics, A J SABHARWAL Royal Hospital for Sick Children, Yorkhill, Glasgow G3 8S7
1. Ladd WE, Gross RE. Congenital diaphragmatic hernia. $N$ Engl f Med 1940;223:917-23.

2 Stolar C, Dillon P, Reyes C. Selective use of extracorporeal membrane oxygenation in the management of congenital diaphragmatic hernia. $\mathcal{F}$ Pediatr Surg 1988;23:207-11

3 Langham Jr MR, Kays DW, Ledbetter DJ, et al. Congenital diaphragmatic hernia: epidemiology and outcome. Clin Perinatol 1996;23:671-88.

4 Dommergues M, Louis-Sylvestere C, Mandelbrot L, et al. Congenital diaphragmatic hernia: can prenatal ultrasonography predict outcome? $\mathrm{Am}$ f Obstet Gynecol 1996;174:1377-81.

5 Sweed Y, Puri P. Congenital diaphragmatic hernia: influence of associated malformations on survival. Arch Dis Child 1994;70:F159.

6 Harrison MR, Adzick NS, Longaker MT, et al. Successful repair in utero of a fetal diaphragmatic hernia after removal of herniated viscera from the left thorax. N Engl f Med 1990;322:1582-4.

7 DiFiore JW, Fauza DO, Slavin R, et al. Experimental fetal tracheal ligation reverses the structural and physiological effects of pulmonary hypoplasia in reverses the structural and physiological effects of pulmonary hyp

8 Hedrick MH, Estes JM, Sullivan KM, et al. Plug the lung until it grows (PLUG): a new method to treat congenital diaphragmatic hernia in utero. (PLUG): a new method to treat $1994 ; 29: 612-17$

9 Harrison MR, Adzick NS, Flake AW, et al. Correction of congenital diaphragmatic hernia in utero: VI. Hard-earned lessons. $\mathcal{F}$ Pediatr Surg 1993;28:1411-18

10 Suen HC, Losty P, Donahoe PK, Schnitzer JJ. Combined antenatal thyrotropin-releasing hormone and low-dose glucocorticoid therapy improves the pulmonary biochemical immaturity in congenital diaphragmatic hernia. $\mathcal{F}$ Pediatr Surg 1994;29:359-63.

11 Senzaki H, Tamura M, Sakakihara Y, Hishi T. A new protocol for the perinatal management of patients with congenital diaphragmatic hernia with severe hypoplastic lungs and its clinical application. Acta Paediatr fpn 1994;36:497-500.

12 Wung JT, Sahni R, Moffitt ST, et al. Congenital diaphragmatic hernia: survival treated with very delayed surgery, spontaneous respiration and no chest tube. $\mathcal{F}$ Pediatr Surg 1995;30:406-9.

13 Azarow K, Pearl R, Filler RM, et al. Congenital diaphragmatic hernia-a tale of two cities: the Toronto experience. F Pediatr Surg 1997;32:395-400.

14 Wilson JM, Thompson JR, Schnitzer JJ, et al. Intratracheal pulmonary ventilation and congenital diaphragmatic hernia: a report of two cases. $\mathcal{F}$ Peditilation and congenital diapt
atr Surg 1993;28:484-7.

15 Kinsella JP, Neish SR, Dunbar D, et al. Clinical responses to prolonged treatment of persistent pulmonary hypertension of the newborn with low treatment of persistent pulmonary hypertension of the
doses of inhaled nitric oxide. F Pediatr 1993;123:103-8.

16 Finer NM, Ehrenkranz RA, Solimano A, et al. Inhaled nitric oxide and hypoxic respiratory failure in infants with congenital diaphragmatic hernia. Pediatrics 1997;99:838-45.

17 Wilcox DT, Glick PL, Karamanoukian HL, et al. Pathophysiology of congenital diaphragmatic hernia XII: amniotic fluid lecithin/ sphingomyelin ratio and phosphatidylglycerol concentrations do not predict surfactant status in congenital diaphragmatic hernia. 7 Pediatr Surg 1995;30:410-12.

18 Lotze A, Knight GR, Anderson KD, et al. Surfactant (beractant) therapy for infants with congenital diaphragmatic hernia on ECMO: evidence of persistent surfactant deficiency. F Pediatr Surg 1994;29:407-12.

19 UK Collaborative ECMO Trial Group. UK collaborative trial of neonatal extracorporeal membrane oxygenation. Lancet 1996,348:75-82.

20 Newman KD, Anderson KD, VanMeurs K, et al. Extracorporeal membrane oxygenation and congenital diaphragmatic hernia: should any infant be oxygenation and congenital diaphragmatic

21 Lally KP, Paranka MS, Roden J, et al. Congenital diaphragmatic hernia. Stabilization and repair on ECMO. Ann Surg 1992;216:569-73.

22 Pranikoff T, Gauger PG, Hirschl RB. Partial liquid ventilation in newborn patients with congenital diaphragmatic hernia. $f$ Pediatr Surg 1996;31:613-18.

23 Van Meurs KP, Rhine WD, Benitz WE, et al. Lobar lung transplantation as a treatment for congenital diaphragmatic hernia. $f$ Pediatr Surg 1994;29:1557-60.

24 Sakai $\mathrm{H}$, Tamura M, Hosokowa Y, et al. Effect of surgical repair on respiratory mechanics in congenital diaphragmatic hernia. $\mathcal{F}$ Pediatr 1987;111:432-8.

25 Stolar CJH. What do survivors of congenital diaphragmatic hernia look like when they grow up? Semin Pediatr Surg 1996;5:275-9. 